\title{
The advanced imaging-guided approach to acute ischemic stroke in the extended reperfusion time window
}

\author{
Andy Lim ${ }^{1,3}$, Channa Senanayake ${ }^{2,3}$, Benjamin Clissold ${ }^{2,3}$, Thanh Phan ${ }^{2,3}$, Henry $\mathrm{Ma}^{2,3}$ \\ 'Department of Emergency Medicine, Monash Medical Centre, Melbourne 3168, Australia. \\ ${ }^{2}$ Department of Neurology, Monash Medical Centre, Melbourne 3168, Australia. \\ ${ }^{3}$ School of Clinical Sciences, Monash University, Melbourne 3800, Australia.
}

Correspondence to: Prof. Henry Ma, Department of Neurology, Monash Medical Centre, 246 Clayton Road, Victoria 3168, Australia. E-mail: henry.ma@monashhealth.org

How to cite this article: Lim A, Senanayake C, Clissold B, Phan T, Ma H. The advanced imaging-guided approach to acute ischemic stroke in the extended reperfusion time window. Vessel Plus 2021;5:34. https://dx.doi.org/10.20517/25741209.2021 .44

Received: 9 Mar 2021 First Decision: 30 Apr 2021 Revised: 5 May 2020 Accepted: 18 May 2021 Published: 23 Jun 2021

Academic Editor: Elisa Francesca Maria Ciceri Copy Editor: Xi-Jun Chen Production Editor: Xi-Jun Chen

\begin{abstract}
Evaluation and treatment of acute ischemic stroke has undergone significant advancement since the 1990s, when acute systemic reperfusion with intravenous alteplase became the first approved method for achieving reperfusion. Until recently, $4.5 \mathrm{~h}$ after stroke onset appeared to be the maximum time window in which positive results from thrombolysis can be achieved. However, the advent of advanced imaging modalities, including multimodal magnetic resonance imaging and computed tomography perfusion has allowed clinicians to refine the evaluation of these patients by delineating areas of infarcted tissue, areas of potentially salvageable tissue given timely reperfusion, and areas of benign oligemia. Early work in extending the time window beyond this historical limit of $4.5 \mathrm{~h}$ has culminated in four positive trials that demonstrate the benefit of acute mechanical or systemic reperfusion therapy in an extended time window of up to $24 \mathrm{~h}$, using advanced imaging criteria for patient selection. The implications of the success of advanced imaging suggest use of these modalities for disposition decisions and selection of greater numbers of patients for reperfusion but add complexity to individual patient evaluation. Despite this, many questions remain unanswered, including the best choice of thrombolysis agent, whether we can extend the time window further to $24 \mathrm{~h}$, and the optimal combination of mechanical thrombectomy and bridging therapy in the late time window in patients with or without large vessel occlusion.
\end{abstract}


Keywords: Stroke, reperfusion, thrombectomy, tissue plasminogen activator, fibrinolytic agents, computed tomography, magnetic resonance imaging

\section{INTRODUCTION}

The evaluation and treatment of acute ischemic stroke has advanced rapidly in the last three decades. New knowledge in reperfusion timing and techniques, advanced imaging, and improved patient selection continues to translate to better outcomes. Early paradigms regarding time-based approaches to treatment have been challenged by positive trials that use advanced imaging criteria to select patients for reperfusion ${ }^{[1-4]}$. In this paper, we will review the initial studies that introduced the clinical efficacy of systemic reperfusion, a brief history of the penumbra and its imaging, and the four seminal trials. We will then explore the implications and clinical benefit of these trials, introduce an alternative application of imaging-guided selection to transient ischemic attack and minor stroke, and outline future direction.

\section{TIME-BASED TARGET OF THROMBOLYSIS}

The first major advance in acute reperfusion therapy occurred in the 1990s when alteplase (rrtPA) became the first approved drug to treat acute ischemic stroke. At the time, the National Institute of Neurological Disorders and Stroke rt-PA Stroke Study Group demonstrated that, despite a small increased incidence of symptomatic intracranial haemorrhage, treatment with $\mathrm{rtPA}$ within $3 \mathrm{~h}$ of onset of symptoms improved clinical outcome $e^{[5]}$. This represented the beginning of a line of clinical inquiry to increase the time window of therapy. Further success came in 2008 when the ECASS $\mathrm{III}^{[6]}$ trial demonstrated the safety and efficacy of alteplase administered between 3 and $4.5 \mathrm{~h}$ after stroke onset. The pooled analyses of clinical trials ${ }^{[7,8]}$ suggested a loss of efficacy after this $4.5 \mathrm{~h}$ mark. It is likely that the choice of non-contrast computed tomography prevented the use of rtPA beyond this $4.5 \mathrm{~h}$ window. Such imaging modality does not capture the presence of salvageable ischemic tissue (ischemic penumbra) nor vessel occlusion. Methods for imaging the ischemic penumbra will be explored in the next section.

\section{THE PENUMBRA AND THE EVOLUTION OF BRAIN IMAGING}

Since the 1970s, early mammalian experiments have noted that when a major artery of the brain was occluded, a rate of cerebral blood flow could be reached that preserved neuronal structure but produced functional inactivity ${ }^{[9]}$. Neurons could actually survive in this state of functional incapacity, and then be normalized again if cerebral blood flow was restored ${ }^{[9]}$. Such a cerebral blood flow zone between the upper threshold of electrical failure and the lower threshold of energy and pump failure was defined as the ischemic penumbra ${ }^{[10]}$. Beyond this zone is a region of hypoperfusion but normal cerebral function experiencing benign oligemia ${ }^{[9]}$. Hence, this early classical model demonstrated that there was a zone of electrical silence in ischemic cerebral tissue that remained potentially salvageable.

There are several modalities for imaging the ischemic penumbra including positron emission tomography (PET), magnetic resonance imaging (MRI) and computed tomography perfusion $(\mathrm{CTP})^{[11]}$. While PET with ${ }^{15} \mathrm{O}$ tracer is regarded as the gold standard method to produce quantitative measurements ${ }^{[12]}$, PET is unfortunately best suited as a research tool rather than use in routine clinical practice ${ }^{[11]}$. Using the hypoxic tissue ligand FMISO $\left({ }^{18} \mathrm{~F}\right.$-fluoromisonidazole) ischemic penumbral tissue was demonstrated to exist for up to $48 \mathrm{~h}$ from stroke onset and its survival was associated with improved functional outcome ${ }^{[13]}$. The early 1990s saw researchers experimenting with dynamic MRI studies that compared perfusion-weighted imaging (PWI) and diffusion-weighted imaging (DWI) to image the ischemic penumbra. PWI measures cerebral blood flow (CBF), cerebral blood volume (CBV), mean transit time (MTT), time to peak (TTP), and delay in tracer arrival $\left(\mathrm{T}_{\max }\right)$. 
The DWI lesion was thought initially to represent the region of restricted diffusion with failure of the $\mathrm{Na}^{+} / \mathrm{K}^{+}$pump. Subsequently, the DWI lesion was found to represent both infarcted tissue and penumbra, and visible perfusion abnormality also included regions of benign oligemia ${ }^{[14]}$. These concepts have been further refined: the current method of estimating infarcted tissue with MRI is by applying an apparent diffusion coefficient threshold of $\leq 620 \times 10^{-6} \mathrm{~mm}^{2} / \mathrm{s}$ to identify core voxels ${ }^{[15]}$. The revised definition of hypoperfused tissue is now $\mathrm{T}_{\max }<6 \mathrm{~s}^{[16]}$. An issue with the use of MR imaging to define the ischemic penumbra is that the DWI and PWI images are acquired at different orientations. As such the mismatch may be underestimated when using the formula PWI lesion minus DWI lesion. The so-called hidden mismatch is revealed when the PWI and DWI images are registered to the same coordinates ${ }^{[17,18]}$. CTP can produce perfusion maps with the same parameters as PWI. It has an advantage in that registration of the images is not required in the calculation of the perfusion mismatch. CTP appears to be a viable alternative and has been demonstrated to have similar accuracy ${ }^{[19,20]}$.

\section{EARLY WORKS}

Both the Echoplanar Imaging Thrombolytic Evaluation Trial (EPITHET) and the Diffusion and Perfusion Imaging Evaluation For Understanding Stroke Evolution (DEFUSE) studies demonstrated favorable outcomes in stroke patients with a perfusion/diffusion mismatch treated with IV rtPA within 3-6 h of symptom onset ${ }^{[2,22]}$. It is thought that the original EPITHET outcome was negative as it included oligemic region in the analysis ( $\mathrm{T}_{\max }$ threshold $2 \mathrm{~s}$ ). In subsequent analysis, changing the perfusion threshold $\left(\mathrm{T}_{\max } 6 \mathrm{~s}\right)$ resulted in positive trial outcome $\mathrm{e}^{[16,23]}$. Furthermore, post-hoc analysis of the combined EPITHET-DEFUSE dataset with the revised $\mathrm{T}_{\max }$ threshold of $>6 \mathrm{~s}$ demonstrated reduced infarct growth and increased reperfusion rates in the mismatch patients who received alteplase ${ }^{[24]}$.

CT perfusion was also available at the time, but investigators were reading it visually, not quantitatively. The double-blind, randomised, placebo-controlled phase 3 trial DIAS-3 (safety and efficacy of desmoteplase given 3-9 h after ischemic stroke in patients with occlusion or high-grade stenosis in major cerebral arteries) demonstrated no improvement in functional outcome with image-guided desmoteplase treatment ${ }^{[25]}$. This visual rather than quantitative analysis of perfusion data may be one of the reasons for the negative result, as it has been demonstrated that volumetric calculation of mismatch is superior to visual assessment ${ }^{[26]}$. Given that visual assessment overestimates tissue at risk of infarction, there was a need for standardization of lesion segmentation using objective criteria to strengthen methodology of further randomized trials. Fully automated software packages were developed which were well validated in estimating ischemic core and penumbra $^{[27,28]}$.

With this in mind, the EXtending the time for Thrombolysis in Emergency Neurological Deficits (EXTEND) trial ${ }^{[20]}$ was designed, building on these lessons. The investigators used automated perfusion software (RAPID, iSchemaView) to aid treatment decision. The purpose was to test the use of perfusion imaging selection of patients who presented 4.5 to $9 \mathrm{~h}$ after stroke onset or with wake-up stroke within $9 \mathrm{~h}$ from the midpoint of sleep ${ }^{[29]}$. Concurrently, a Multicenter, Randomized, Double-Blind, Placebo-Controlled Trial to Test Efficacy and Safety of Magnetic Resonance Imaging-Based Thrombolysis in Wake-up Stroke (WAKE-UP), was designed to test whether a different imaging approach (DWI-FLAIR mismatch) could be used to select stroke patients with unknown time of onset e.g., waking up with symptoms ${ }^{[30]}$.

Two endovascular thrombectomy trials also attempted to demonstrate the clinical benefit from selecting patients with advanced imaging for endovascular clot retrieval in the late time window. The DAWN trial (DWI or CTP Assessment With Clinical Mismatch in the Triage of Wake-Up and Late Presenting Strokes Undergoing Neurointervention With Trevo) tested the concept of clinical-imaging mismatch to select their 
patients $^{[2]}$. The DEFUSE-3 (Endovascular Therapy Following Imaging Evaluation for Ischemic Stroke) trial chose to use CT or MR penumbral imaging ${ }^{[1]}$. All these lines of inquiry have culminated into four seminal trials that demonstrate the benefit of image-guided patient selection for reperfusion, and this will be explored in the next section.

\section{POSITIVE TRIALS}

Four recent positive trials provide evidence for acute mechanical ${ }^{[1,2]}$ and systemic ${ }^{[3,4]}$ reperfusion therapy in an extended time window up to $24 \mathrm{~h}$, using advanced imaging criteria for patient selection.

\section{EXTEND}

The EXTEND trial selected patients based on penumbral imaging 4.5 to $9 \mathrm{~h}$ after stroke onset or with wakeup stroke within $9 \mathrm{~h}$ from the midpoint of sleep. A total of 225 patients were enrolled, with 113 receiving alteplase and 112 receiving placebo. The primary outcome (modified Rankin Scale) of 0-1 occurred in 35.4\% in the alteplase group and $29.5 \%$ in the placebo group ${ }^{[3]}$. This meant there was a $44 \%$ higher likelihood of a good outcome in the alteplase group compared to placebo - ARR 1.44 (1.01-2.06), $P=0.04^{[3]}$. In other words, alteplase was shown to improve functional outcomes at 3 months when administered 4.5 to $9 \mathrm{~h}$ or after wake-up stroke less than $9 \mathrm{~h}$ from midpoint of sleep amongst patients with perfusion mismatch. This was a consistent effect across age, time, and large vessel strata. It is notable that in EXTEND 69\% of patients in the alteplase group had large vessel occlusion with a recanalization rate at $24 \mathrm{~h}$ of $67.3 \%$ without mechanical thrombectomy ${ }^{[3]}$. This made EXTEND the first positive trial of intravenous thrombolysis in patients in the extended time window selected with penumbral imaging ${ }^{[31]}$. It is also important to recognise that a significant number of patients in EXTEND had wake up stroke.

\section{WAKE-UP}

The WAKE-UP trial selected stroke patients with unknown time of onset using selection based on DWIFLAIR mismatch, with DWI estimating ischemia and FLAIR estimating infarct. The investigators assumed that the absence of FLAIR signal indicated that the ischemic injury occurred within $4.5 \mathrm{~h}$ of onset. After enrolment of 503 patients, 254 received alteplase and 249 received placebo; a favorable outcome (modified Rankin Scale of 0-1) was achieved in 53\% of the alteplase group and $42 \%$ of the placebo group [adjusted odds ratio $(\mathrm{OR})=1.61 ; 95 \%$ confidence interval $(\mathrm{CI}): 1.09-2.36 ; P=0.02]^{[4]}$. The rate of symptomatic intracranial hemorrhage did not differ significantly, with $2.0 \%$ in the alteplase group and $0.4 \%$ in the placebo group $(\mathrm{OR}=4.95 ; 95 \% \mathrm{CI} \text { : 0.57-42.87; } P=0.15)^{[4]}$. Such a finding then led to the termination of several other trials that were investigating a similar concept due to a loss of equipoise $e^{[3,32,33]}$. This then became the first randomized evidence of intravenous thrombolysis in patients with unknown time of symptoms guided by advanced imaging $^{[31]}$.

\section{DAWN and DEFUSE-3}

The DAWN trial tested the concept of clinical-imaging mismatch to select patients for mechanical reperfusion in the $6-24 \mathrm{~h}^{[2]}$. The algorithm for clinical-imaging mismatch was complicated and based on age, stroke severity and infarct volume. Infarct volume was defined on CT or DWI using automated software (RAPID, iSchemiaView). For patients $\geq 80$-year-old and NIHSS $\geq 10$, the infarct volume had to be less than $21 \mathrm{~mL}$. For patients $<80$-year-old and NIHSS $\geq 10$, the infarct volume had to be less than $31 \mathrm{~mL}$. For patients $<80$-year-old and NIHSS $\geq 20$, the infarct volume had to be between 31-51 mL. Patients selected using this method demonstrated significantly better functional outcome at 90 days $^{[2]}$. Alternatively, the DEFUSE-3 trial employed penumbral imaging up to 6-16 h from onset to become the first randomized evidence of reperfusion benefit when acute stroke patients with an extended or unknown time window were selected with penumbral imaging ${ }^{[3]}$. Note that the time of onset of stroke for these two trials was calculated 
from the time the patient was last known to be well, in contrast to the EXTEND definition of wake-up stroke measured from the midpoint of sleep.

\section{Other studies}

At the same time as EXTEND trial, ECASS-4 also evaluated perfusion imaging of the penumbra for thrombolysis ${ }^{[33]}$. The THAWS trial ${ }^{[32]}$ used the same DWI-FLAIR mismatch concept as WAKE-UP trial. These trials were terminated early following the WAKE-UP results. Individually, they did not demonstrate a difference in favorable outcome between alteplase and control groups ${ }^{[2,33]}$. This is likely a reflection of these studies lacking sufficient statistical power due to the relatively low number of recruited patients to demonstrate the desired effect (ECASS-4119 patients recruited with planned 264 patients and THAWS 131 patients recruited with planned 300 patients). MR WITNESS was a phase $2 \mathrm{a}$, open-label safety trial of intravenous thrombolysis in stroke patients with unwitnessed symptom onset within 4.5 to $24 \mathrm{~h}$ time window. The trial is different from WAKE-UP which used visual analysis of DWI-FLAIR mismatch. MR WITNESS tested quantified DWI-FLAIR mismatch (qDFM) as imaging selection criteria. The results were encouraging with regards to the use of qDFM to guide therapy. Eighty patients were recruited with 39\% achieving mRS 0-1 at 90 days while only one patient sustained symptomatic intracranial hemorrhage ${ }^{[34]}$.

\section{Pooled results}

A meta-analysis of EXTEND, ECASS4, and EPITHET provided strong evidence of efficacy for patients with an unknown time of onset or in the extended time window ${ }^{[35]}$. Note that this was pooled individual data, much more powerful than a standard meta-analysis. Another research group independently confirmed these findings with their meta-analysis of EXTEND, WAKE-UP and ECASS $4^{[36]}$. A further meta-analysis of combined EXTEND, WAKE-UP, ECASS4, and THAWS data demonstrated a strong benefit of thrombolysis $v s$. placebo for independent functional outcome for the unclear time of onset subset ${ }^{[37]}$.

\section{Guideline adaptation}

The Australian Clinical Guidelines for Stroke Management ${ }^{[38]}$ has strongly recommended that for patients with potentially disabling ischemic stroke who meet perfusion mismatch criteria in addition to standard clinical criteria, intravenous alteplase (dose of $0.9 \mathrm{mg} / \mathrm{kg}$, maximum of $90 \mathrm{mg}$ ) should be administered up to $9 \mathrm{~h}$ after the time the patient was last known to be well, or from the midpoint of sleep for patients who wake with stroke symptoms, unless immediate endovascular thrombectomy is planned ${ }^{[38]}$. The American Heart Association/American Stroke Association guidelines were last updated in $2019^{[39]}$ and prior to publication of the EXTEND trial. The European Stroke Organisation (ESO) had already released a consensus statement that IV alteplase may be considered for patients with acute ischemic stroke 4.5 to $9 \mathrm{~h}$ from onset with a penumbral mismatch back in $2018^{[40]}$. This has recently been formalized, with the group stating that "for patients with ischemic stroke of 4.5 to $9 \mathrm{~h}$ duration (known onset time) and with CT or MRI core/perfusion mismatch, and for whom mechanical thrombectomy is either not indicated or not planned, we recommend intravenous thrombolysis with alteplase" ${ }^{[41]}$.

\section{UNANSWERED QUESTIONS} Implementation

Implementing the DWI-FLAIR protocol requires access to MR scanner. This may not be possible outside of tertiary teaching hospitals. By contrast, multimodal CT scanners are available even in rural parts of Australia and can be combined with automated perfusion analysis ${ }^{[27]}$. An advantage of DWI-FLAIR mismatch over multimodality CT is that it can be used in patients with significant renal impairment. Further it can be read visually and without investment in expensive software. It can be argued that to achieve the trial results of EXTEND and DEFUSE3, investment in software such as RAPID is required. The hospitals involved in these trials have experience with using RAPID software and can recognize false 
positive cases. As such it is not certain if the results of the trials can easily be replicated without the aid of an experienced stroke physician or radiologist ${ }^{[42]}$.

\section{Minor ischaemic stroke}

The use of perfusion imaging to aid alteplase therapy in patients with minor ischemic stroke is unclear. Minor ischemic stroke is often defined as an NIHSS less than or equal to 5. Clinical trials of alteplase in patients with minor stroke have not used advanced imaging. This was the case with the negative PRISM trial ${ }^{[43]}$. It is possible that advanced imaging may help to find an enriched cohort who may benefit from reperfusion therapy. An observational study demonstrated that mismatch between hypoperfused tissue and ischemic core in high risk TIA and minor ischemic stroke is predictive of neurological deterioration, infarct progression, and disability ${ }^{[4]]}$. This concept has not yet been tested in clinical trial.

\section{Is alteplase the best choice of thrombolysis agent?}

Unanswered questions remain about the optimal choice of thrombolysis agent in the early window. Although some evidence exists for tenecteplase in perfusion-selected patients within 6 h of onset ${ }^{[45]}$ or with large vessel occlusion within $4.5 \mathrm{~h}^{[46]}$, the best choice remains unanswered. The NOR-TEST trial ${ }^{[47]}$ demonstrated that tenecteplase was not superior to alteplase in the early window, but its non-inferiority is yet to be answered. The ongoing TASTE trial (NCT04071613) is addressing the comparison between early intravenous tenecteplase and intravenous alteplase within $4.5 \mathrm{~h}$ of stroke onset using perfusion imaging selection.

\section{Does systemic reperfusion work up to $24 \mathrm{~h}$ after stroke onset?}

The penumbra can remain viable for up to $48 \mathrm{~h}$ after stroke onset in selected patients ${ }^{[48]}$. Studies such as this one suggest that thrombolysis beyond $9 \mathrm{~h}$ is possible. Two trials are currently recruiting to address this. The ETERNAL trial (NCT04454788) seeks to answer whether reperfusion of penumbral tissue with tenecteplase in patients with large vessel occlusion is beneficial. This phase III, prospective, randomized, open-label, blinded endpoint design is evaluating patients presenting to the emergency department with a hyperacute stroke due to large vessel occlusion who are eligible for thrombectomy, and who have a perfusion mismatch. Patients will be randomized to receive tenecteplase or standard care before clot retrieval. TIMELESS (NCT03785678) is a phase III, prospective, double blind, randomized, placebo-controlled trial that seeks to test the efficacy and safety of tenecteplase in patients with a large vessel occlusion (internal carotid artery or middle cerebral artery) and penumbral tissue within 4.5 to $24 \mathrm{~h}$. The primary outcome will be the ordinal modified Rankin Scale score at day 90.

\section{What is the best way to reperfuse in the late time window in patients with or without large vessel occlusion?}

In patients with large vessel occlusion, the utility of direct thrombectomy or bridging therapy with intravenous thrombolysis followed by thrombectomy within the late time window, is being studied. The DIRECT MT trial demonstrated non-inferiority of direct thrombectomy compared to bridging therapy within $4.5 \mathrm{~h}$ of stroke onset. However, this study utilized non-contrast CT for patient selection ${ }^{[49]}$. There are a number of ongoing trials attempting to answer the same question, including DIRECT-SAFE (NCT03494920) and MR CLEAN-NO IV (ISRCTN80619088) trials. For the same question but in the beyond $4.5 \mathrm{~h}$ time window, the ETERNAL and TIMELESS trials will, hopefully, provide the answers. In patients with large vessel occlusion who are ineligible for thrombectomy, whether intravenous thrombolysis will prove to be superior to placebo for functional improvement is another area of considerable interest. The final difficult patient group are those without large vessel occlusion in the late time window. 


\section{Can infarct and penumbral location aid clinical decision?}

Up till now, the concept of the ischemic penumbra has been discussed in terms of mismatch volume and without information on location ${ }^{[1,50]}$. Such information can help to reveal why some patients with large ischemic core have recovery of motor function. This latter observation of good outcome despite having a large core requires an understanding of the topography of the penumbra and the function of the infarcted regions. Analysis taking into account information on neuroanatomical location of penumbra and infarct requires complex multivariate analysis and is not yet available for clinical use.

\section{CONCLUSION}

Much progress has been made in the evaluation of acute stroke patients with advanced imaging. Randomized trial evidence now exists that demonstrates positive functional outcome when patient selection is based on advanced imaging, challenging the previous paradigm of time-based stroke therapies. The recent success of this tissue-based approach is set to influence current stroke management and the future direction of stroke research.

\section{DECLARATIONS}

Authors' contributions

Involved in the conception, literature review, editing and final approval: Lim A, Senanayake C, Clissold B, Phan T, Ma H

Involved in the drafting of the initial manuscript: Lim A

\section{Availability of data and materials}

Not applicable.

\section{Financial support and sponsorship}

Lim A is supported by an Australian Government Research Training Program (RTP) Scholarship and is in receipt of the Monash Health Emerging Researcher Fellowship.

\section{Conflicts of interest}

All authors declared that there are no conflicts of interest.

\section{Ethical approval and consent to participate}

Not applicable.

\section{Consent for publication}

Not applicable.

\section{Copyright}

(c) The Author(s) 2021.

\section{REFERENCES}

1. Albers GW, Marks MP, Kemp S, et al. Thrombectomy for stroke at 6 to 16 hours with selection by perfusion imaging. New Engl $J$ Med 2018;378:708-18. DOI PubMed PMC

2. Nogueira RG, Jadhav AP, Haussen DC, et al. Thrombectomy 6 to 24 hours after stroke with a mismatch between deficit and infarct. New Engl J Med 2018;378:11-21. DOI PubMed

3. Ma H, Campbell BCV, Parsons MW, et al. Thrombolysis guided by perfusion imaging up to 9 hours after onset of stroke. $N$ Engl $J$ Med 2019;380:1795-803. DOI PubMed

4. Thomalla G, Simonsen CZ, Boutitie F, et al; WAKE-UP Investigators. MRI-guided thrombolysis for stroke with unknown time of onset. N Engl J Med 2018;379:611-22. DOI PubMed

5. Institute of Neurological Disorders Stroke rt-PA Stroke Study Group. Tissue plasminogen activator for acute ischemic stroke. New Engl J Med 1995;333:1581-7. DOI PubMed 
6. Hacke W, Kaste M, Bluhmki E, et al. Thrombolysis with alteplase 3 to 4.5 hours after acute ischemic stroke. New Engl J Med 2008;359:1317-29. DOI PubMed

7. Emberson J, Lees KR, Lyden P, et al. Effect of treatment delay, age, and stroke severity on the effects of intravenous thrombolysis with alteplase for acute ischaemic stroke: a meta-analysis of individual patient data from randomised trials. Lancet 2014;384:1929-35. DOI PubMed PMC

8. Lees KR, Emberson J, Blackwell L, et al. Effects of alteplase for acute stroke on the distribution of functional outcomes. Stroke 2016;47:2373-9. DOI PubMed PMC

9. Astrup J, Symon L, Branston NM, Lassen NA. Cortical evoked potential and extracellular K+ and $\mathrm{H}+$ at critical levels of brain ischemia. Stroke 1977;8:51-7. DOI PubMed

10. Astrup J, Siesjö BK, Symon L. Thresholds in cerebral ischemia - the ischemic penumbra. Stroke 1981;12:723-5. DOI PubMed

11. Thirugnanachandran T, Ma H, Singhal S, et al. Refining the ischemic penumbra with topography. Int J Stroke 2018;13:277-84. DOI PubMed

12. Davis S, Donnan GA. Time is penumbra: imaging, selection and outcome. Cerebrovasc Dis 2014;38:59-72. DOI PubMed

13. Markus R, Reutens DC, Kazui S, et al. Hypoxic tissue in ischaemic stroke: persistence and clinical consequences of spontaneous survival. Brain 2004;127:1427-36. DOI PubMed

14. Kidwell CS, Alger JR, Saver JL. Beyond mismatch: evolving paradigms in imaging the ischemic penumbra with multimodal magnetic resonance imaging. Stroke 2003;34:2729-35. DOI PubMed

15. Purushotham A, Campbell BCV, Straka M, et al. Apparent diffusion coefficient threshold for delineation of ischemic core. Int J Stroke 2015;10:348-53. DOI PubMed PMC

16. Olivot JM, Mlynash M, Thijs VN, et al. Optimal Tmax threshold for predicting penumbral tissue in acute stroke. Stroke 2009;40:46975. DOI PubMed PMC

17. Ma HK, Zavala JA, Churilov L, et al. The hidden mismatch: an explanation for infarct growth without perfusion-weighted imaging/diffusion-weighted imaging mismatch in patients with acute ischemic stroke. Stroke 2011;42:662-8. DOI PubMed

18. Ma H, Zavala JA, Teoh H, et al. Penumbral mismatch is underestimated using standard volumetric methods and this is exacerbated with time. J Neurol Neurosurg Psychiatry 2009;80:991-6. DOI PubMed

19. Campbell BC, Christensen S, Levi CR, et al. Comparison of computed tomography perfusion and magnetic resonance imaging perfusion-diffusion mismatch in ischemic stroke. Stroke 2012;43:2648-53. DOI PubMed

20. Wintermark M, Reichhart M, Cuisenaire O, et al. Comparison of admission perfusion computed tomography and qualitative diffusionand perfusion-weighted magnetic resonance imaging in acute stroke patients. Stroke 2002;33:2025-31. DOI PubMed

21. Davis SM, Donnan GA, Parsons MW, et al. Effects of alteplase beyond $3 \mathrm{~h}$ after stroke in the Echoplanar Imaging Thrombolytic Evaluation Trial (EPITHET): a placebo-controlled randomised trial. Lancet Neurol 2008;7:299-309. DOI PubMed

22. Albers GW, Thijs VN, Wechsler L, et al; DEFUSE Investigators. Magnetic resonance imaging profiles predict clinical response to early reperfusion: the diffusion and perfusion imaging evaluation for understanding stroke evolution (DEFUSE) study. Ann Neurol 2006;60:508-17. DOI PubMed

23. Picanço MR, Christensen S, Campbell BC, et al. Reperfusion after 4.5 hours reduces infarct growth and improves clinical outcomes. Int J Stroke 2014;9:266-9. DOI PubMed

24. Ogata T, Christensen S, Nagakane Y, et al. The effects of alteplase 3 to 6 hours after stroke in the EPITHET-DEFUSE combined dataset. Stroke 2013;44:87-93. DOI PubMed

25. Albers GW, von Kummer R, Truelsen T, et al. Safety and efficacy of desmoteplase given 3-9 h after ischaemic stroke in patients with occlusion or high-grade stenosis in major cerebral arteries (DIAS-3): a double-blind, randomised, placebo-controlled phase 3 trial. Lancet Neurol 2015;14:575-84. DOI PubMed

26. Campbell BC, Christensen S, Foster SJ, et al; EPITHET Investigators. Visual assessment of perfusion-diffusion mismatch is inadequate to select patients for thrombolysis. Cerebrovasc Dis 2010;29:592-6. DOI PubMed

27. Campbell BC, Parsons MW. Imaging selection for acute stroke intervention. Int J Stroke 2018;13:554-67. DOI PubMed

28. Campbell BC, Yassi N, Ma H, et al. Imaging selection in ischemic stroke: feasibility of automated CT-perfusion analysis. Int J Stroke 2015;10:51-4. DOI PubMed

29. Ma H, Parsons MW, Christensen S, et al. A multicentre, randomized, double-blinded, placebo-controlled Phase III study to investigate EXtending the time for Thrombolysis in Emergency Neurological Deficits (EXTEND). Int J Stroke 2012;7:74-80. DOI PubMed

30. Thomalla G, Fiebach JB, Østergaard L, et al. A multicenter, randomized, double-blind, placebo-controlled trial to test efficacy and safety of magnetic resonance imaging-based thrombolysis in wake-up stroke (WAKE-UP). Int J Stroke 2014;9:829-36. DOI PubMed

31. Thomalla G, Gerloff C. Acute imaging for evidence-based treatment of ischemic stroke. Curr Opin Neurol 2019;32:521-9. DOI PubMed

32. Koga M, Yamamoto H, Inoue M, et al; THAWS Trial Investigators. Thrombolysis with alteplase at $0.6 \mathrm{mg} / \mathrm{kg}$ for stroke with unknown time of onset. Stroke 2020;51:1530-8. DOI PubMed PMC

33. Ringleb P, Bendszus M, Bluhmki E, et al. Extending the time window for intravenous thrombolysis in acute ischemic stroke using magnetic resonance imaging-based patient selection. Int J Stroke 2019;14:483-90. DOI PubMed

34. Schwamm LH, Wu O, Song SS, et al. Intravenous thrombolysis in unwitnessed stroke onset: MR WITNESS trial results. Ann Neurol 2018;83:980-93. DOI PubMed PMC

35. Campbell BC, Ma H, Ringleb PA, et al; EXTEND; ECASS-4; and EPITHET Investigators. Extending thrombolysis to 4.5-9 h and wake-up stroke using perfusion imaging: a systematic review and meta-analysis of individual patient data. Lancet 2019;394:139-47.

DOI PubMed 
36. Tsivgoulis G, Katsanos AH, Malhotra K, et al. Thrombolysis for acute ischemic stroke in the unwitnessed or extended therapeutic time window. Neurology 2020;94:e1241. DOI PubMed

37. Thomalla G, Boutitie F, Ma H, et al; Evaluation of unknown Onset Stroke thrombolysis trials (EOS) investigators. Intravenous alteplase for stroke with unknown time of onset guided by advanced imaging: systematic review and meta-analysis of individual patient data. Lancet 2020;396:1574-84. DOI PubMed PMC

38. . Stroke Foundation. Clinical Guidelines for Stroke Management.

39. Powers WJ, Rabinstein AA, Ackerson T, et al. Guidelines for the early management of patients with acute ischemic stroke: 2019 update to the 2018 Guidelines for the early management of acute ischemic stroke: a Guideline for healthcare professionals from the American Heart Association/American Stroke Association. Stroke 2019;50:e344-418. DOI PubMed

40. Ahmed N, Audebert H, Turc G, et al. Consensus statements and recommendations from the ESO-Karolinska Stroke Update Conference, Stockholm 11-13 November 2018. Eur Stroke J 2019;4:307-17. DOI PubMed PMC

41. Berge E, Whiteley W, Audebert H, et al. European Stroke Organisation (ESO) guidelines on intravenous thrombolysis for acute ischaemic stroke. Eur Stroke J 2021;6:I-LXII. DOI PubMed PMC

42. Leira EC, Muir KW. EXTEND trial: towards a more inclusive but complex thrombolysis. Stroke 2019;50:2637-9. DOI PubMed

43. Khatri P, Kleindorfer DO, Devlin T, et al. Effect of alteplase vs aspirin on functional outcome for patients with acute ischemic stroke and minor nondisabling neurologic deficits: the PRISMS randomized clinical trial. JAMA 2018;320:156-66. DOI PubMed PMC

44. Asdaghi N, Hill MD, Coulter JI, et al. Perfusion MR predicts outcome in high-risk transient ischemic attack/minor stroke: a derivationvalidation study. Stroke 2013;44:2486-92. DOI PubMed

45. Parsons M, Spratt N, Bivard A, et al. A randomized trial of tenecteplase versus alteplase for acute ischemic stroke. $N$ Engl J Med 2012;366:1099-107. DOI PubMed

46. Campbell BC, Mitchell PJ, Churilov L, et al. Tenecteplase versus alteplase before thrombectomy for ischemic stroke. N Engl J Med 2018;378:1573-82. DOI PubMed

47. Logallo N, Novotny V, Assmus J, et al. Tenecteplase versus alteplase for management of acute ischaemic stroke (NOR-TEST): a phase 3, randomised, open-label, blinded endpoint trial. Lancet Neurol 2017;16:781-8. DOI PubMed

48. Ma H, Wright P, Allport L, et al. Salvage of the PWI/DWI mismatch up to $48 \mathrm{~h}$ from stroke onset leads to favorable clinical outcome. Int J Stroke 2015;10:565-70. DOI PubMed

49. Yang P, Zhang Y, Zhang L, et al; DIRECT-MT Investigators. Endovascular thrombectomy with or without intravenous alteplase in acute stroke. N Engl J Med 2020;382:1981-93. DOI PubMed

50. Phan TG, Chen J, Donnan G, Srikanth V, Wood A, Reutens DC. Development of a new tool to correlate stroke outcome with infarct topography: a proof-of-concept study. Neuroimage 2010;49:127-33. DOI PubMed 